\title{
Shortest Path Problem under Fuzziness
}

Luhandjula MK $^{*}$ and Strydom MC

Department of Decision Sciences, University of South Africa, P O Box 392, Unisa, Pretoria 0003, South Africa

*Corresponding author: Luhandjula MK, Department of Decision Sciences, University of South Africa, South Africa, Tel: 0124334660; E-mail: luhanmk@unisa.ac.za Rec date: Dec 16, 2013; Acc date: Feb 25, 2014; Pub date: Apr 30, 2014

Copyright: (c) 2014 Luhandjula MK, et al. This is an open-access article distributed under the terms of the Creative Commons Attribution License, which permits unrestricted use, distribution, and reproduction in any medium, provided the original author and source are credited.

\begin{abstract}
The shortest path problem plays a paramount role in a wide spectrum of domains including transportation, communication and networking. The "primum movens" of this paper is to propose a novel approach for solving the shortest path problem with fuzzy parameters. Two key issues need to be addressed in a way to handle the fuzzy path problem. One is how to determine the sum of two edges. The other is how to compare the lengths of two different paths given that the length of each edge is represented by fuzzy numbers.

To solve these problems we resort to the notions of "nearest interval approximation" and average of a fuzzy number.An assessment of our approach, in comparison with extant ones is also provided. A Numerical example is given for the sake of illustration.
\end{abstract}

Keywords: Fuzzy numbers; Shortest path; Nearest interval

\section{Introduction}

Many non-trivial real life decision problems, including those that may be cast into the shortest path problem footing, are hardly modeled in deterministic terms because of uncertainty or incompleteness of related information.

In this connection, the British economist Shackle [1] was saying: "In a predestinate world, decision would be illusory; in a world of perfect for e knowledge, empty; in a world without order, powerless. Our intuitive attitude to life implies non-illusory, non-empty, nonpowerless decision.

Since decision in this sense excludes both perfect foresight and anarchy in nature, it must be defined as choice in face of bounded uncertainty". [2]

This has also been expressed by Zadeh's incompatibility principle

"When the complexity of a system increases, our ability to formulate precise and yet meaningful statements decreases up to a threshold beyond which precision and significance become mutually exclusive characteristics.

The need for mathematical models under uncertainty is then obvious.

In this paper we propose a new approach for handling the shortest path problem with fuzzy parameters.

Our approach is based on the notions of "nearest interval approximation and average of a fuzzy number.

Open Rubric: It contrasts markedly with those based on defuzzification operators which replace a fuzzy set by a single real number leading to a loss of much important information.
The challenging task of singling out the shortest path out of the resulting deterministic problem with interval lengths is also addressed.

The remaining of the paper unfolds as follows; Section 2 is devoted to preliminary materials that are needed in the sequel. In section 3 , we describe our approach for solving the shortest path problem under fuzziness on a sequential graph.

We end up in Section 4 with some concluding remarks along with lines for further developments in this field.

\section{Preliminaries}

\section{Real intervals}

We denote by Kc , the family of all bounded closed intervals in $1 \mathrm{R}$; i.e.,

$$
K c=\{[a, b] \mid a, b \varepsilon \mathbb{R} \text { and } a \leq b\}
$$

For $A \varepsilon K c$, we write $A=\left[a^{L}, a^{U}\right]$ where aL and aU are respectively the lower and the upper bounds of $A$. The centre and the width of the interval $\mathrm{A}=[\mathrm{aL}, \mathrm{aU}]$ are respectively given by ${ }_{a} C=\frac{1}{2}\left[a^{L},{ }^{L} U\right]$, $a^{S}=a^{U}-a^{L}$

The main idea behind interval arithmetic is as follows:

Let $* \in\{+,-, \times, \div\}$ be a binary operation in $\mathbb{R}$. If $A$ and $B$ belong to

Kc, then

$A * B=\{a * b \mid a \in A$ and $b \in B\}$

Defines a binary operation on Kc. 
Page 2 of 6

In the case of division, it is assumed that 0 is not a member of interval B. Arithmetic operations on intervals used in this paper, may be explicitly obtained from (1) as follows.

For A, B E Kc we have:

$A+B=\left[a^{L}, a^{U}\right]+\left[b^{L}+b^{U}\right]=\left[a^{L}+b^{L}, a^{U}+b^{U}\right]$

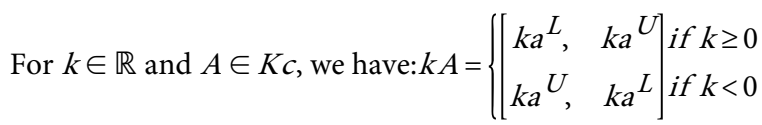

The following are some order relations defined on $\mathrm{Kc}$ [3].

Let $A=\left[a^{L}, a^{U}\right]$ and $B=\left[b^{L}, b^{U}\right]$

The order relation $\leq L U$ is defined as follows: $A \leq L U$ B if and only if $a^{L} \leq b^{L}$ and $a{ }^{U} \leq b^{U}$

The order relations $\leq L S$ and $\geq L S$ are defined as follows: $A \leq L S$ B if and only if ${ }^{L} \leq b^{L}$ and $a^{S} \leq b^{S}$

$A \geq L S B$ if and only if $a{ }^{U} \leq b^{U}$ and $a^{S} \leq b^{S}$

The order relation $\leq$ is defined as follows $A \leq B$ if and only if $R$ $R$ ${ }_{a}^{U} \leq b^{L}$

\section{Fuzzy numbers}

A fuzzy subset $\bar{a}$ of the real line $\mathbb{R}$ with membership function: $u \bar{a}: \mathbb{R} \rightarrow[0,1]$ is called a fuzzy number if the following conditions hold. 1. $\bar{a}$ is normal, i.e. there is $x_{0} \in \mathbb{R}$. Such that $\mu \bar{a}\left(x_{0}\right)=1$

$\mu \tilde{a}\left(\lambda x 1+(1-\lambda) x_{2}\right) \geq \min \left(\mu \tilde{a}\left(x_{1}\right), \mu \tilde{a}\left(x_{2}\right)\right)$ for $x 1, x 2 \in \mathbb{R}, \lambda \in[0,1]$.

Supp $\bar{a}=\frac{}{\{x \in \mathbb{R} \mid \mu \bar{a}(x)>0\}}$ is bounded.

A useful tool for dealing with a fuzzy number is its $\alpha$-cut.

The a-cut of a fuzzy number $\bar{a}$ is the crisp set defined as follows.

$\tilde{a}_{\alpha}=\{x \in \mathbb{R} \mid \mu \tilde{a}(x) \geq \alpha\}$. According to the above definition of a fuzzy number, every a -cut of a fuzzy number is a closed interval. Therefore, in the sequel $\bar{a}_{\alpha}$ will be written as $\left[\bar{a}_{\alpha} L, \bar{a}_{\alpha} U\right]$

Where, $\quad \bar{a}_{\alpha} L=\inf \{x \in \mathbb{R} \mid \mu \tilde{a}(x) \geq \alpha\}$ and

$\bar{a}_{\alpha} U=\sup \{x \in \mathbb{R} \mid \mu \tilde{a}(x) \geq \alpha\}$

For more details on fuzzy numbers, the reader may consult [4]. In this paper, the space of fuzzy numbers of is denoted by $F(\mathbb{R})$. In the sequel we'll also use the notion of a core of a fuzzy number. The core of $\bar{a}$ denoted by $\operatorname{core}(\bar{a})$ is defined as follows: Core $(\bar{a})=\{x \in \mathbb{R} \mid \mu \tilde{a}(x)=1\}$

In $\operatorname{space} F(\mathbb{R})$, a metric has been defined as follows.For $\tilde{a}, \tilde{b} \in F(\mathbb{R})$

$$
d_{f}(\mathbb{R})(\tilde{a}, \tilde{b})=\sup _{\alpha \in[0,1]} d_{H\left(\tilde{a}_{\alpha}, \tilde{b}_{\alpha}\right)}
$$

where $\mathrm{dH}$ stands for the Hausdorff metric. In this paper we'll use triangular and trapezoidal fuzzy numbers, i.e. fuzzy numbers having the membership functions given in Figures 1 and 2.

\section{Nearest interval approximation of a fuzzy number}

An interval approximation of a fuzzy number is an operator

$C: F(\mathbb{R}) \rightarrow K c$ Such that for $\tilde{a} \in F(\mathbb{R})$

1. $C(\tilde{a}) \subset \sup p \tilde{a}$

2. $\operatorname{Core}(\tilde{a}) \subset C(\tilde{a})$

3. $\forall \in>0, \exists \delta>0 \mid d_{F} f(\mathbb{R})(\tilde{a}, \tilde{b})<\delta$

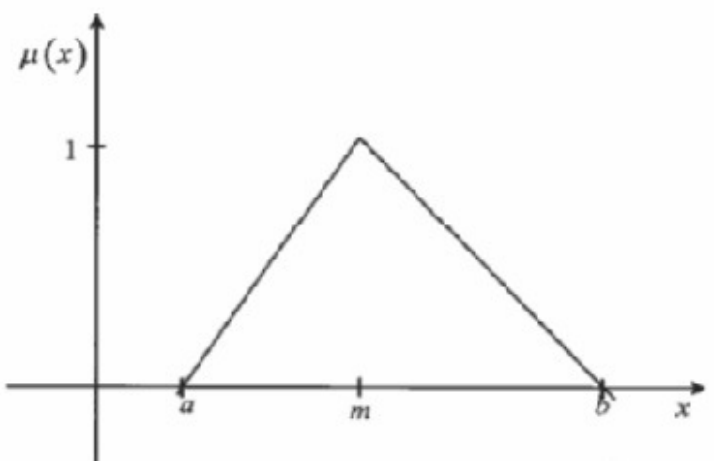

Figure 1: Triangular fuzzy number denoted by ${ }^{\sim} \mathrm{m} \Delta(\mathrm{m}, \mathrm{a}, \mathrm{b})$

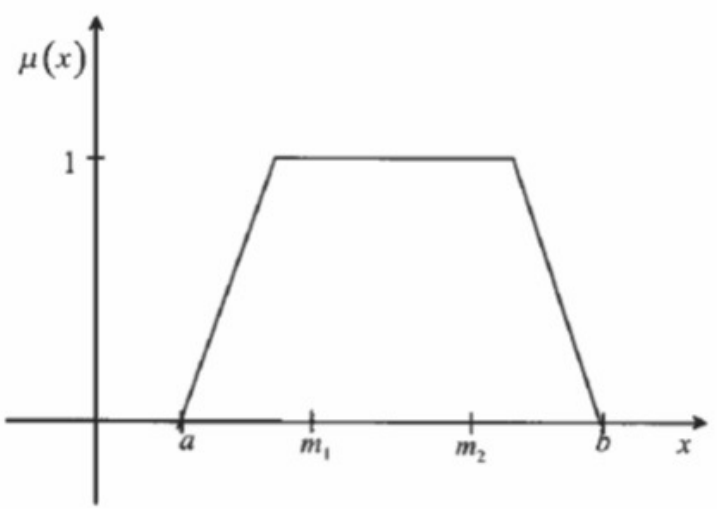

Figure 2: Trapezoidal fuzzy number denoted by $\sim \mathrm{m}=\Delta(\mathrm{m} 1, \mathrm{~m} 2$, a, b) $\Rightarrow H(C(\tilde{a}), C(\tilde{b}))<\varepsilon$

A nearest interval approximation (NIA) of a fuzzy number $\bar{a}$ with respect to the metric $d F(\mathbb{R})$ is an interval approximation of $\bar{a}, C d(\bar{a})$ that minimized $F(\mathbb{R})(\bar{a}, C(\bar{a}))$.

For all $\mathrm{C}$ in the space of interval approximation operators of fuzzy numbers

This means for any interval approximation $C(\bar{a})$, the following relation holds. $d_{F(\mathbb{R})}\left(C_{d}(\bar{a}), \bar{a}\right) \leq d F(\mathbb{R})(C(\bar{a}), \bar{a})$. In what precedes, an interval $[\mathrm{a}, \mathrm{b}]$ is considered as a fuzzy number $\bar{a}$ whose membership function is the indicator of $[\mathrm{a}, \mathrm{b}]$ i.e., $\mu_{\bar{a}}(x)= \begin{cases}1 & \text { if } x \in[a, b] \\ 0 & \text { otherwise }\end{cases}$

The question that comes to mind is how to find the nearest interval approximation of a fuzzy number. 
Page 3 of 6

An answer to this question is given by the following result.

\section{Proposition}

Consider a fuzzy number $\bar{a}$ and define the following functions: $f^{L}:[0,1] \rightarrow \mathbb{R}$

$$
\begin{aligned}
\alpha & \rightarrow f^{L}(\alpha)=\bar{a}_{\alpha} L \\
f^{U}:[0,1] & \rightarrow \mathbb{R} \\
\alpha & \rightarrow f^{U}(\alpha)=\bar{a}_{\alpha} U
\end{aligned}
$$

Where

$$
\bar{a}^{L} \alpha=\inf \left\{x \in \mathbb{R} \mid \mu_{\alpha}(x) \geq \alpha\right\}
$$

$\bar{a} U_{\alpha}=\sup \{x \in \mathbb{R} \mid \mu \bar{\alpha}(x) \geq \alpha\}$

$$
\text { then } C_{d}(\bar{a})=\left[\int_{0}^{1} f^{L}(\alpha) d \alpha, \int_{0}^{1} f^{U}(\alpha) d \alpha\right.
$$

For the proof of this result, the reader may consult [ 3].

\section{Finding a shortest path in a sequential graph with fuzzy values}

\section{Problem formulation}

We assume that the values of edges are either triangulars.

Consider the sequential graph given in Figure 3.

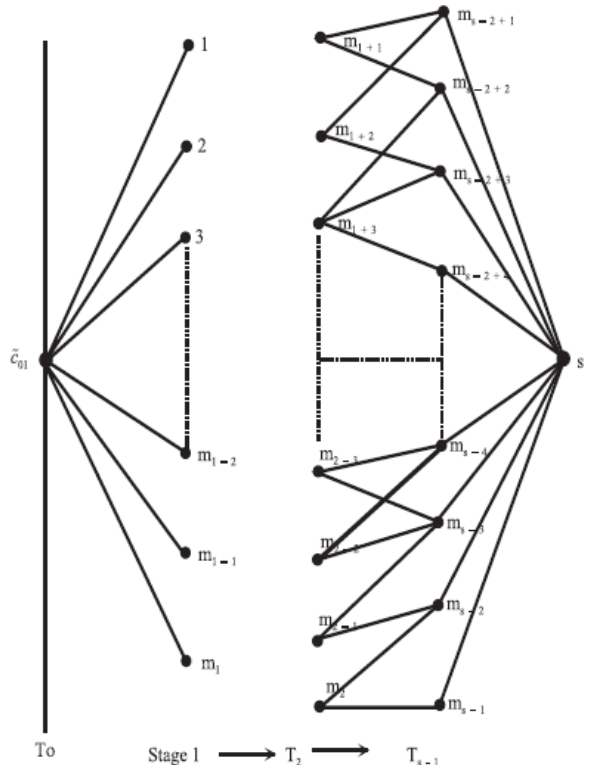

Figure 3: A sequential graph

In this figure, $T_{0}=\{0\}$ and $T_{S}=\{s\}$

Moreover we have $T_{1}=\left\{1,2, \ldots \ldots ., m_{1}\right\}$

$T_{2}=\left\{m_{1}+1, \ldots . ., m_{2}\right\}$, and more generally, $T_{j}=\left\{m_{j}-1, \ldots, m_{j}\right\}$. We denote by $\bar{c}_{i j}$ the length of the path between $i$ and $j$ and by $M$ the number of edges in the graph. We suppose that in a way to couple their subjective perceptions with huge statistical data, decision makers prefer to present $\bar{c}_{i j}$ fuzzy numbers.For convenience, the values (lengths) of edge are numbered as follows $\bar{e}_{p}, p=1, \ldots ., M$

This means that $\bar{e}_{1}$ denotes the first value, $\bar{e}_{2}$ the second value etc. The problem is to find the path with the shortest length.

\section{Solution method}

Principle: The main idea behind our approach is to approximate involved fuzzy numbers by their nearest interval approximations (NIA). The NIA of these fuzzy numbers are small intervals as there are included in the supports of corresponding fuzzy numbers. The resulting shortest path problem with interval edge values is then solved using the Bellman principle.

We make use of interval arithmetic [5] (see also \$2) to find the sum of intervals.

Regarding the comparison of intervals, we proceed as follows.

First the most stringent order relation $\leq$ is defined in $\$ 2$, is $R$

considered.If this relation cannot discriminate intervals at hand, we consider $\leq$ (see $\$ 2$ ). If this relation cannot discriminate interval under 2

consideration we resort to the order relation $\frac{<}{3}$ that is justified because of our assumption on the sizes of supports of involved fuzzy numbers. It is worth noticing that for disjoint intervals $\frac{<}{2}$ and $\frac{<}{3}$ boil down to $\frac{<}{1}$

From the foregoing we can devise an algorithm for solving the fuzzy shortest path problem for a sequential graph.

Description of the algorithm: This algorithm proceeds in 3 steps.

Step 1: Find the NIA of involved fuzzy numbers.

Step 2: Apply forwardly or backwardly the Bellman principle to find the minimal length denoted by $v_{o s}^{*}$

This is done step by step. If we opt for the forward option, for instance,

We should find first $v_{o j}^{*} \forall j \in T_{\wedge}$ and then $v_{o l}^{*} \forall I \in T_{2}, \ldots v_{o n}^{*} \forall \xi \in T_{S-1}$

And finally $v_{o s}^{*}$

Step 3: Determine the path from the initial node 0 to the final note $s$ that provides the minimal length $v_{o s}^{*}$

\section{Oracles used in the proposed algorithm:}

The following 3 oracles, the flow charts of which are given below, are used in an essential manner in our algorithm (Figures 4-7). 


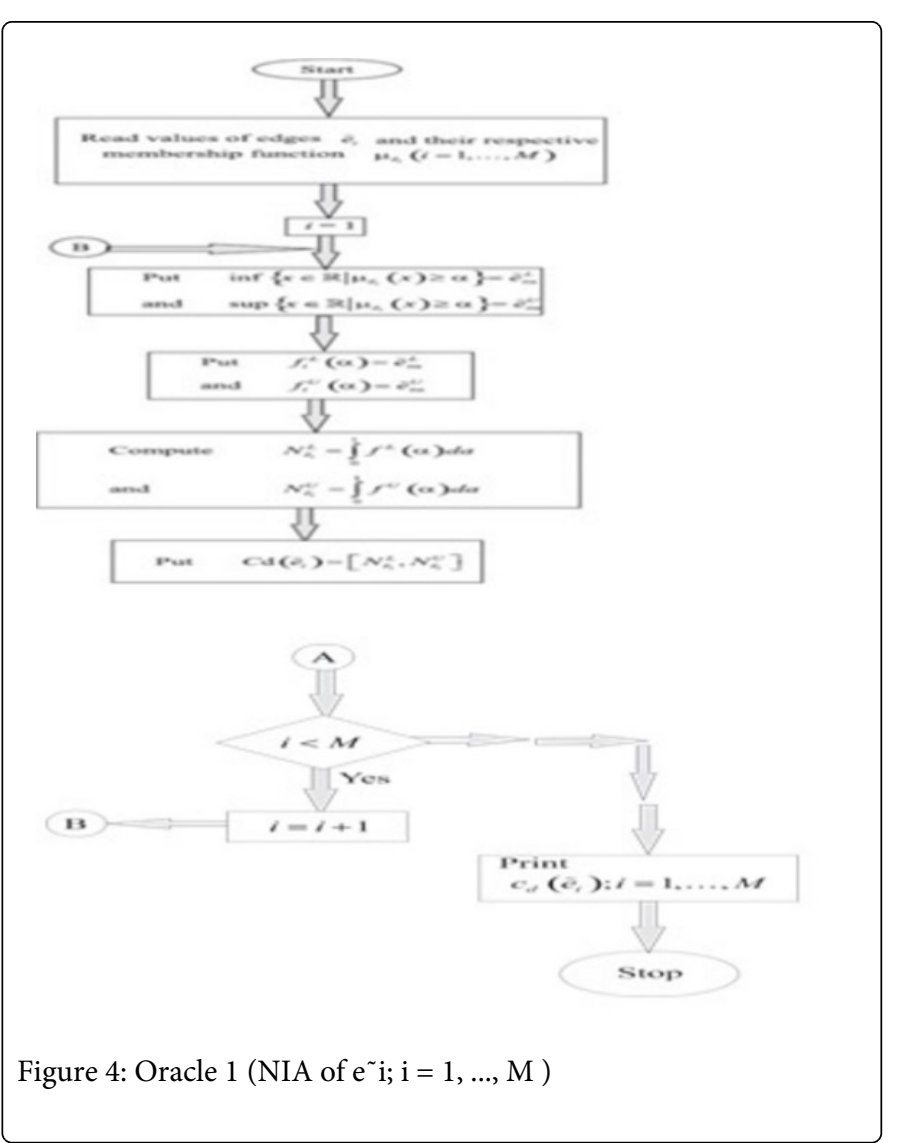

\section{Flowchart of the proposed algorithm}

\section{Numerical example}

Problem formulation: Consider the following sequential graph with triangular fuzzy numbers values of edges as defined in Figure 6(a)-(f) The values represent costs.

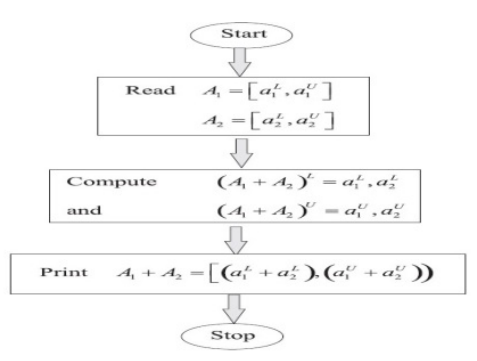

Figure 5: Oracle 2 (Sum of two intervals)

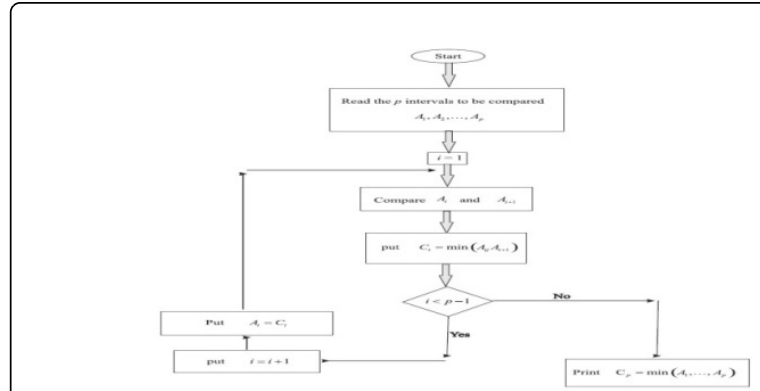

Figure 6: Oracle 3 (Find the min of p intervals A1 , A2 , .., Ap )

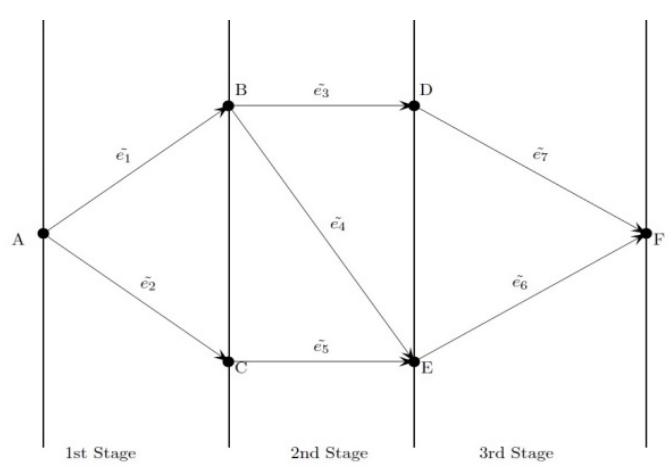

Figure 7: Here $M=7$ and we have 3 stages.

\section{Solving the problem}

The nearest interval approximation (NIA) of each $\bar{e}_{j}(j=1,2 \ldots .6)$ has been computed, using the formula obtained in proposition (Figures $8-13)$. These interval approximations are given in (Table 1).

\begin{tabular}{|l|l|}
\hline Edge value & Corresponding NIA \\
\hline$€ \mathrm{t}$ & {$[3.75,4.25]$} \\
\hline$€ 2$ & {$[4.75,5.25]$} \\
\hline$€ 3$ & {$[0.75,1.25]$} \\
\hline$€ 4$ & {$[1.9,2.5]$} \\
\hline e5 6 & {$[5.75,6.25]$} \\
\hline & {$[6.75,7.25]$} \\
\hline
\end{tabular}

Table 1: Nearest interval approximation of $\bar{e} j(j=1,2 \ldots .6)$ 


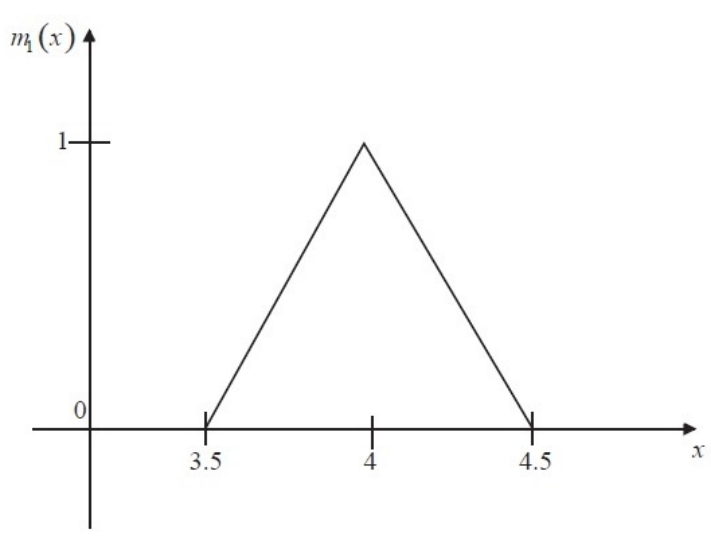

Figure 8: Membership function of $\mathrm{e}^{\sim} 1$

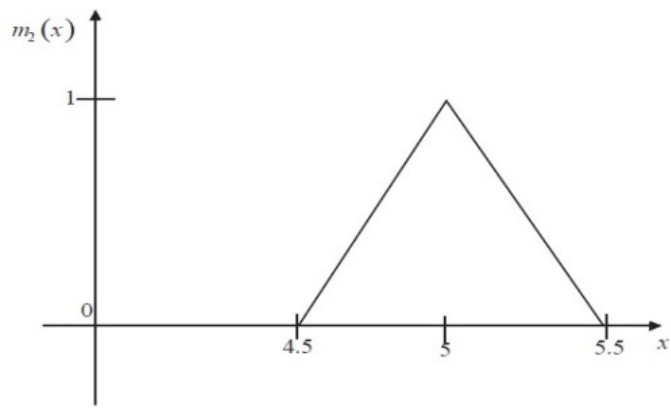

Figure 9: Membership function of $\mathrm{e}^{\sim} 2$

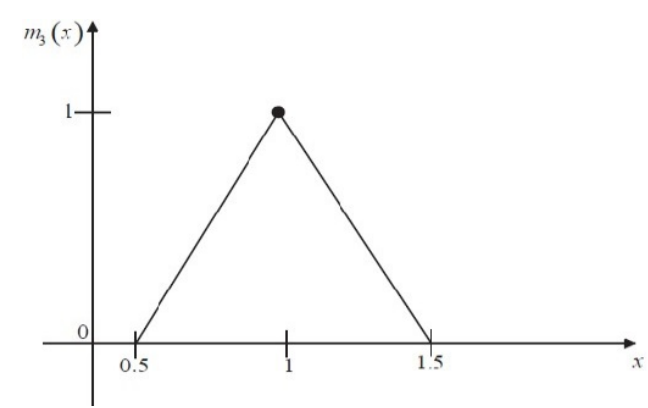

Figure 10: Membership function of $\mathrm{e}^{\sim} 3$
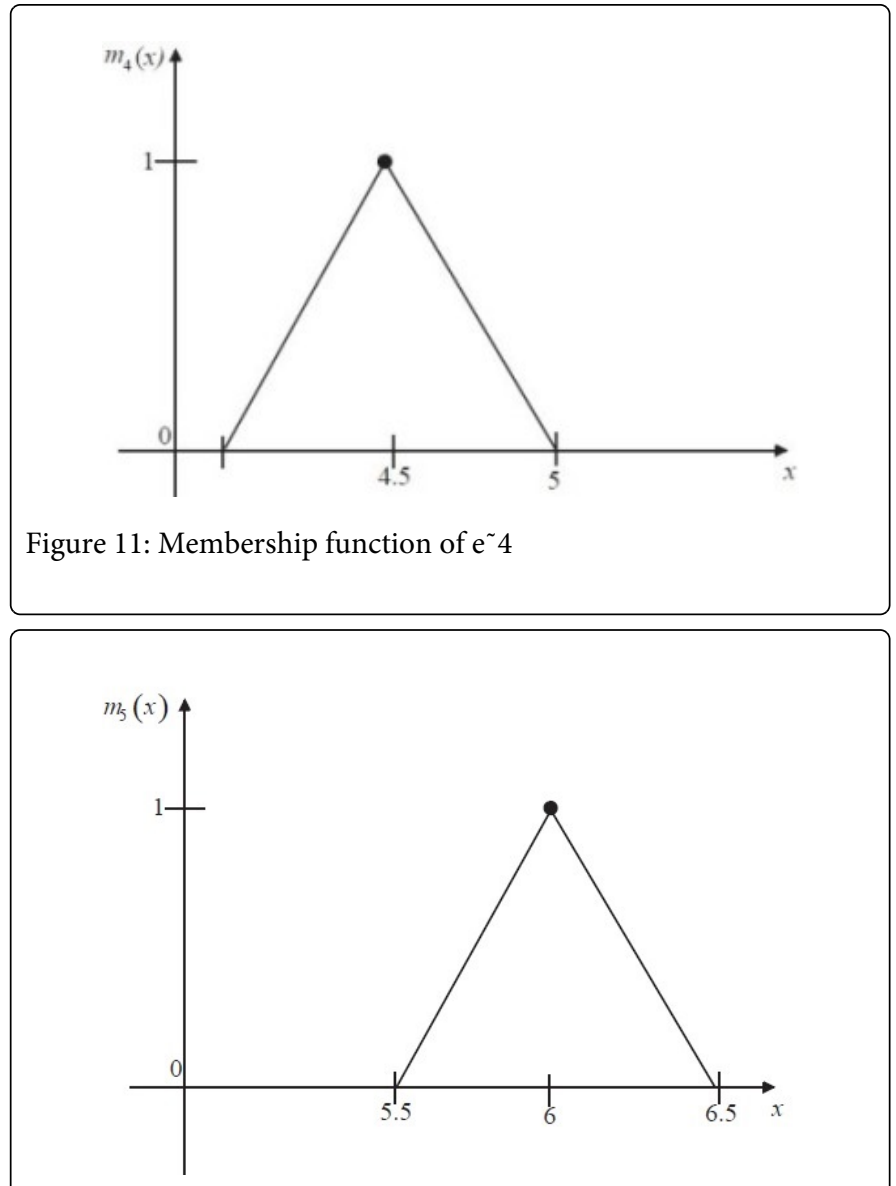

Figure 12: Membership function of $\mathrm{e}^{\sim} 5$

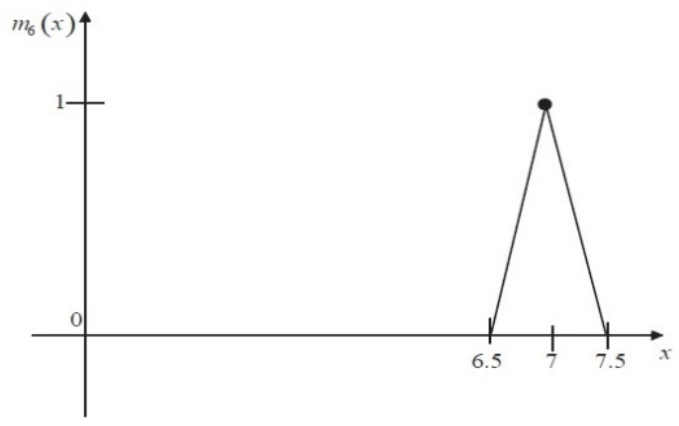

Figure 13: Membership function of $\mathrm{e}^{\sim} 6$

Now we are in a position to apply the proposed algorithm. We use a forward approach. A backward approach can be used as well.

\begin{tabular}{|l|l|l|l|}
\hline 1st stage & & & \\
\hline Node & Path & Cost & Optimal cost \\
\hline B & AB & {$[3.75,4.25]$} & {$[3.75,4.25]$} \\
\hline CB & AC & {$[4.75,5.25]$} & {$[4.75,5.25]$} \\
\hline
\end{tabular}


Page 6 of 6

\begin{tabular}{|l|l|l|l|}
\hline 1st and 2nd stages & & & \\
\hline Node & Path & Cost & Optimal cost \\
\hline D & ABD & {$[4.5,5.5]$} & {$[4.5,5.5]$} \\
\hline E & ABE ACE & {$[5.65,6.75]^{*}[10.5,11.5]$} & {$[5.65,6.75]$} \\
\hline Node & Path & Cost & Optimal cost \\
\hline F & ABDF & {$[9.25,10.75]^{*}$} & \\
\hline E & ABEF & {$[12.45,14.00]^{*}$} & {$[9.25,10.75]$} \\
\hline
\end{tabular}

Table 2: All the 3 stages

Therefore the optimal (minimal path) is ABDF.

Remark: Implementation of this procedure is ongoing and we'll report it, along with numerical experiments, in the near future.

\section{Assessment of our approach}

As can be seen from the literature [6-8] existing approaches for dealing with the shortest path problem under fuzziness are computationally tractable but they do not translate faithfully the original problem. The approach proposed here strikes a balance between effectiveness and efficiency. Effectiveness is due to the fact that involved fuzzy quantities are not re placed by real values (leading to a big loss of information). They replaced by intervals that capture essential features of original fuzzy quantities and that are closest to the corresponding fuzzy quantities. Efficiency is due to the fact that needed operations (sum, comparison) are performed in a deterministic environment rather than an uncertain one. This balance between effectiveness and efficiency gives to our approach a big advantage over existing ones.

\section{Conclusion}

In this paper, we have proposed an approach that cares about both effectiveness and efficiency while dealing with the shortest path problem under fuzziness. Case studies need to be carried out in a way to make transition from theory and practice in this setting. More importantly, numerical experiments should be carried out in a way to compare the performance of our method with other ones. This is the subject matter of a next paper.

Another direction for further development is to generalize our approach to the case of a non-sequential graph. A promising way to deal with this problem is to adapt the Dijkstra algorithm to the case of fuzzy values of edges.

\section{References}

1. Shackle GLS (1961) The Ruin of Economy. Kyklos 14: 482-496.

2. Dubois D, Prade HM (1980) Fuzzy Sets and Systems: Theory and Applications. Academic Press.

3. Ishibushi H, Tanaka H (1990) Multiobjective programming in Optimization of the interval objective function. Eur J Oper Res 48: 219-225.

4. Grzegorewski P (2002) Nearest Interval Approximation of a Fuzzy Number. Fuzzy Set Syst 130: 321-330.

5. Moore RE (1966) Interval Analysis. Prentice-Hall, USA.

6. Schweickerdt GA, Miranda V (2007) A Fuzzy Dynamic Programming Approach for Evaluation of Expansion Distribution Cost in Uncertain Environments. Latin American Applied Research 37: 1-11.

7. Lin FT (2009) Shortest Path Problem Based on Interval- Valued Fuzzy Numbers and Signed Distance Defuzzification Method. Proceedings of 2009 Fourth International Conference on Innovative Computing, Information and Control: 605-608.

8. Klein CM (1991) Fuzzy shortest paths. Fuzzy sets and systems 39: 27-41. 